\title{
Crystal structure of the (REE)-uranyl carbonate mineral shabaite-(Nd)
}

\author{
Jakub PLÁŠIL1, Radek ŠKODA \\ ${ }^{1}$ Institute of Physics, Academy of Sciences of the Czech Republic v.v.i, Na Slovance 2, 18221 Prague 8, Czech Republic; plasil@fzu.cz \\ ${ }^{2}$ Department of Geological Sciences, Masaryk University, Kotlářská 2, 61137 Brno, Czech Republic \\ * Corresponding author
}

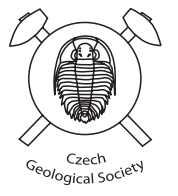

Shabaite-(Nd) is a rare supergene mineral formed during alteration-hydration weathering of uraninite; its structure has remained unknown until now. Based on single-crystal X-ray diffraction data, shabaite-(Nd) is triclinic, twinned (leading to a pseudo-monoclinic diffraction pattern), space group $P$-1, with $a=8.3835(5), b=9.2766(12), c=31.7519(3) \AA, \alpha=$ 90.058(3), $\beta=89.945(4), \gamma=90.331(4)^{\circ}, V=2469.3(4) \AA^{3}$ and $Z=4$. The structure was refined from diffraction data to $R=0.060$ for 8434 unique observed reflections. The structure of shabaite-( $\mathrm{Nd})$ is based upon finite clusters of polyhedra, the well-known uranyl-tricarbonate cluster (referred to as UTC), the Ca-polyhedra linked to UTC and infinite sheets of Nd-polyhedra with internal Nd-O-C linkages. The infinite sheets of Nd-polyhedra are stacked perpendicular to c; to the UTC are staggered approximately perpendicular to the Nd-based sheets (thus approx. parallel to c), forming electroneutral $\left(\left\{\mathrm{Nd}^{3+}{ }_{2}\left(\mathrm{CO}_{3}\right)^{2-}{ }_{2}\left(\mathrm{H}_{2} \mathrm{O}\right)_{2}\right\}^{2+}\left\{\mathrm{Ca}\left(\mathrm{H}_{2} \mathrm{O}\right)_{5}\right\}^{2+}\left[\left(\mathrm{UO}_{2}\right)\left(\mathrm{CO}_{3}\right)_{3}\right]^{4-}\right)^{0}$ layers. Adjacent layers are linked by hydrogen bonds. The ratio of elements in the chemical formula of shabaite-(Nd), obtained from the structure refinement, $\mathrm{Nd}_{2} \mathrm{Ca}\left[\left(\mathrm{UO}_{2}\right)\left(\mathrm{CO}_{3}\right)_{3}\right]$ $\left(\mathrm{CO}_{3}\right)_{2}\left(\mathrm{H}_{2} \mathrm{O}\right)_{10.5}, \mathrm{Z}=4$, was confirmed by an electron-microprobe study. The resulting empirical formula for the average of six analyses is $\mathrm{Ca}_{1.01}\left(\mathrm{Nd}_{0.64} \mathrm{Ce}_{0.32} \mathrm{Sm}_{0.28} \mathrm{Gd}_{0.19} \mathrm{Y}_{0.19} \mathrm{Pr}_{0.13} \mathrm{Dy}_{0.10} \mathrm{La}_{0.07} \mathrm{~Tb}_{0.05} \mathrm{Ho}_{0.01}\right)_{\Sigma 1.98}\left(\mathrm{UO}_{2}\right)\left(\mathrm{CO}_{3}\right)_{4.98}\left(\mathrm{H}_{2} \mathrm{O}\right)_{10.5}$ (based on $1 \mathrm{U}$ $a p f u)$. A discussion of the crystal-structural relationships of shabaite-(Nd) and chemically related minerals is given as well as discussion of complexities of their crystal structures.

Keywords: shabaite-(Nd), uranyl carbonate, rare-earth elements, crystal structure, mineral evolution

Received: 4 October, 2016; accepted: 5 March 2017; handling editor: F. Laufek

The online version of this article (doi: 10.3190/jgeosci.232) contains supplementary electronic material.

\section{Introduction}

Uranyl carbonates are important alteration products resulting from weathering of uraninite, $\mathrm{UO}_{2+\mathrm{x}}$, i.e. hydration-oxidation weathering (Finch and Murakami 1999; Krivovichev and Plášil 2013; Plášil 2014). They are of great environmental importance due to the thermodynamic stability of dissolved uranyl carbonate complexes in aqueous solutions. Uranyl carbonate complexes are often responsible for increased mobility of $\mathrm{U}^{6+}$ (in the form of the uranyl ion, $\mathrm{UO}_{2}{ }^{2+}$ ), in particular under weakly acidic to alkaline conditions (Langmuir 1978). The increased activity of $\mathrm{CO}_{2}$ in solution is due to addition of juvenile $\mathrm{CO}_{2}$, dissolution of common gangue carbonates, or presence of atmospheric $\mathrm{CO}_{2}$ in descending/meteoric waters. Uranyl carbonates most frequently occur as efflorescence on mine walls, adits and tunnels, and as characteristic minerals formed (sub-) recently due to post-mining weathering (Plášil 2014 and references therein).

Of the 34 currently described uranyl carbonates, four or five contain rare-earth elements (REE) as substantial constituents. The difference in total count is due to the recent discovery that kamotoite- $(\mathrm{Y})$ (Deliens and Piret 1986) and bijvoetite-(Y) (Deliens and Piret 1982; Li et al. 2000) most probably are the same species (Plášil and Petř́iček in print). Shabaite-(Nd) was described from the Kamoto East open cut, Shaba province, Katanga, Democratic Republic of Congo by Deliens and Piret (1989). According to the original description, it occurs as micaceous flakes and rosettes on uraninite matrix, in association with other REE-bearing minerals and uranophane. To date, its crystal structure has remained unknown, mainly due to the low quality of available crystals. Here we present the first structure determination based on single-crystal X-ray diffraction data.

\section{Occurrence}

The two samples of shabaite-(Nd) investigated in this study originate from its type locality, Kamoto East open cut (Democratic Republic of Congo). Shabaite-(Nd) is present on both samples as very thin tabular crystals having strong vitreous luster. They are colorless, beige or pale beige in color (Fig. 1). The crystal aggregates have dimensions up to $0.2-0.3 \mathrm{~mm}$ across. Shabaite-(Nd) is associated with astrocyanite-(Ce) and overgrows the strongly altered surface of uraninite. 


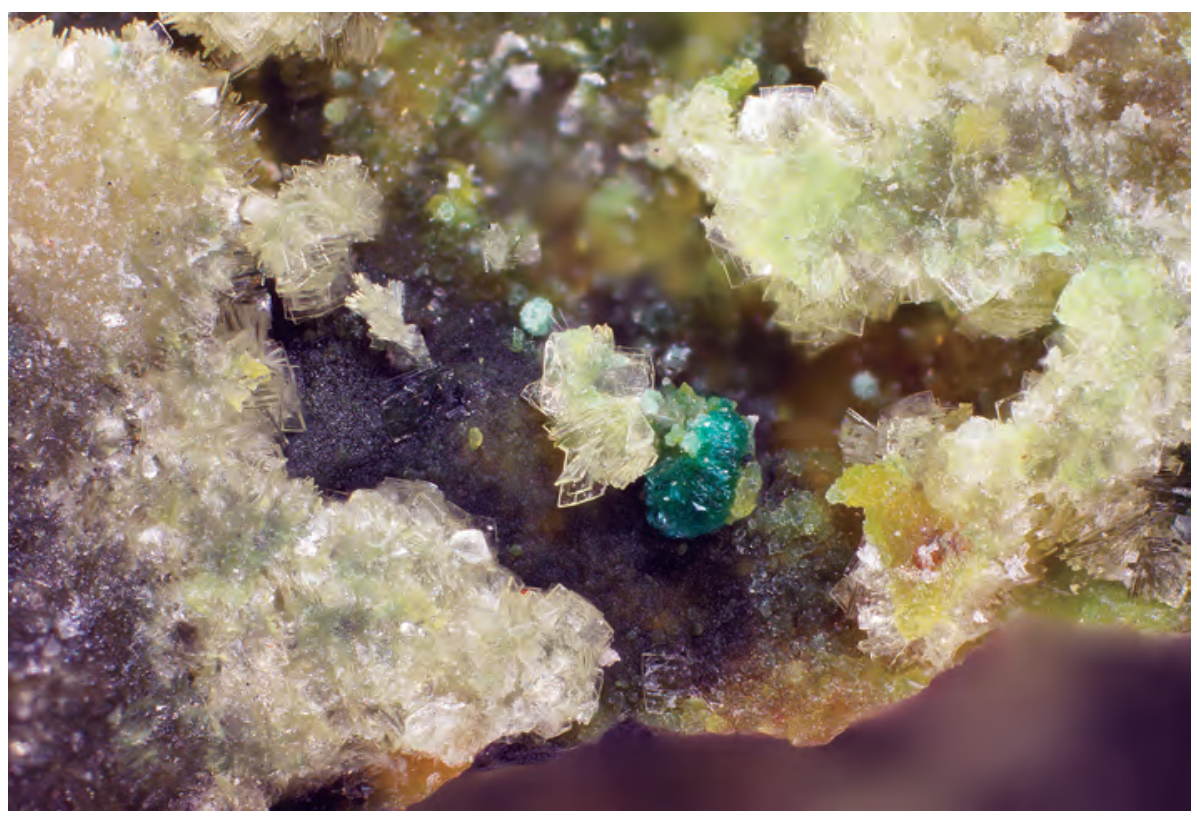

Fig. 1 Pale yellow-whitish tabular crystals of shabaite-(Nd) in association with astrocyanite-(Ce) (bluish-greenish globular aggregate) overgrowing strongly supergene altered uraninite matrix. Width of image is $2.5 \mathrm{~mm}$ (photo P. Škácha).

\section{Chemical composition}

The chemical composition of shabaite-(Nd) was determined using a Cameca SX 100 electron microprobe operated in WDS mode. Two experimental setups were adopted to acquire the quantitative data: 1) for major elements ( $\mathrm{Y}, \mathrm{Nd}, \mathrm{U}$ and $\mathrm{Ca}$ ) an operating voltage of $15 \mathrm{kV}$, beam current of $10 \mathrm{nA}$ and a $8 \mu \mathrm{m}$ beam diameter; 2) for minor elements (REE) beam current of $20 \mathrm{nA}$ was used instead. The following X-ray lines and standards were chosen to minimize line overlaps: $K_{\alpha}$ lines: $\mathrm{Ca}$ (fluorapatite); $L_{\alpha}$ lines: $\mathrm{Y}\left(\mathrm{YPO}_{4}\right), \mathrm{La}\left(\mathrm{LaPO}_{4}\right), \mathrm{Ce}\left(\mathrm{CePO}_{4}\right)$, Dy $\left(\mathrm{DyPO}_{4}\right), \mathrm{Er}\left(\mathrm{ErPO}_{4}\right) ; L_{\beta}$ lines: $\mathrm{Pr}\left(\mathrm{PrPO}_{4}\right), \mathrm{Nd}\left(\mathrm{NdPO}_{4}\right)$, $\mathrm{Sm}\left(\mathrm{SmPO}_{4}\right), \mathrm{Eu}\left(\mathrm{EuPO}_{4}\right), \mathrm{Gd}\left(\mathrm{GdPO}_{4}\right)$, Ho $\left(\mathrm{HoPO}_{4}\right)$; $M_{\beta}$ lines: $\mathrm{U}(\mathrm{U})$. Peak counting times were $10-20 \mathrm{~s}$ and

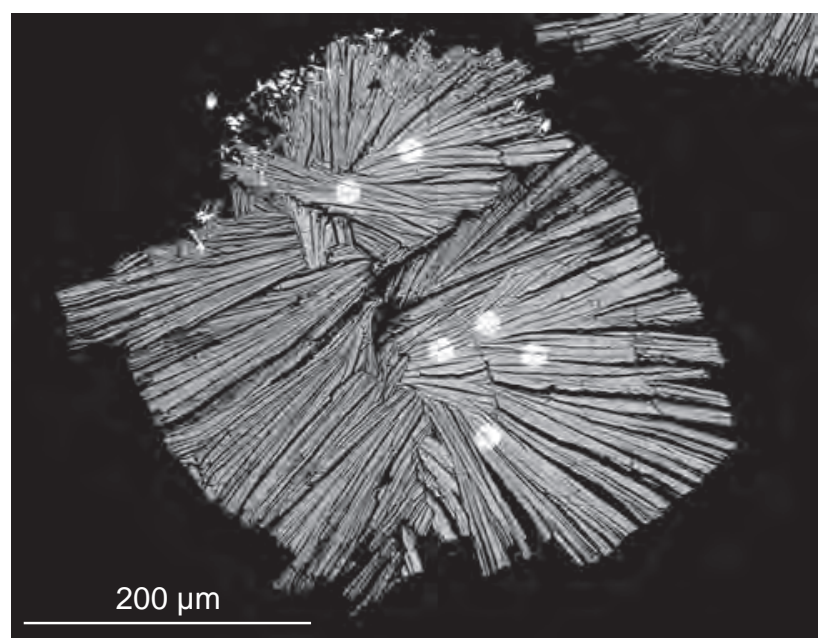

Fig. 2 Aggregate of shabaite-(Nd) in back-scattered electron image showing no apparent chemical zoning of the crystals. Bright spots correspond to analyzed points. the counting time for the background was $50 \%$ of that of the peak. Matrix effects were accounted for using the PAP correction routine (Pouchou and Pichoir 1985). Because of the paucity of pure material for thermal analysis, content of molecular $\mathrm{H}_{2} \mathrm{O}$ was not determined directly, but calculated from stoichiometry obtained by the crystal structure model. On the basis of BSE images, shabaite-(Nd) is chemically homogeneous with no apparent chemical zoning (Fig. 2). The empirical formula, calculated as the mean of 6 representative points (Tab. 1), is $\mathrm{Ca}_{1.01}\left(\mathrm{Nd}_{0.64} \mathrm{Ce}_{0.32} \mathrm{Sm}_{0.28} \mathrm{Gd}_{0.19} \mathrm{Y}_{0.19} \mathrm{Pr}_{0.13} \mathrm{Dy}_{0.10} \mathrm{La}_{0.07} \mathrm{~Tb}_{0.05}\right.$ $\left.\mathrm{Ho}_{0.01}\right)_{\Sigma 1.98}\left(\mathrm{UO}_{2}\right)\left(\mathrm{CO}_{3}\right)_{4.98}\left(\mathrm{H}_{2} \mathrm{O}\right)_{10.5}$ (based on $1 \mathrm{U}$ apfu $)$.

Tab. 1 Results of chemical analyses (in wt. \%) of shabaite-(Nd)

\begin{tabular}{lcccc}
\hline & Mean $(n=6)$ & Range & SD & Standard \\
\hline $\mathrm{CaO}$ & 6.02 & $5.95-6.19$ & 0.09 & wollastonite \\
$\mathrm{Nd}_{2} \mathrm{O}_{3}$ & 11.47 & $10.59-12.26$ & 0.56 & $\mathrm{NdPO}_{4}$ \\
$\mathrm{Ce}_{2} \mathrm{O}_{3}$ & 5.56 & $3.96-6.43$ & 0.85 & $\mathrm{CePO}_{4}$ \\
$\mathrm{Sm}_{2} \mathrm{O}_{3}$ & 5.11 & $4.91-5.48$ & 0.20 & $\mathrm{SmPO}_{4}$ \\
$\mathrm{Gd}_{2} \mathrm{O}_{3}$ & 3.69 & $3.44-4.04$ & 0.21 & $\mathrm{GdPO}_{4}$ \\
$\mathrm{La}_{2} \mathrm{O}_{3}$ & 1.16 & $0.97-1.41$ & 0.19 & $\mathrm{LaPO}_{4}$ \\
$\mathrm{Eu}_{2} \mathrm{O}_{3}$ & 1.39 & $1.25-1.55$ & 0.10 & $\mathrm{EuPO}_{4}$ \\
$\mathrm{Pr}_{2} \mathrm{O}_{3}$ & 2.21 & $2.07-2.36$ & 0.13 & $\mathrm{PrPO}_{4}$ \\
$\mathrm{Dy}_{2} \mathrm{O}_{3}$ & 1.91 & $1.78-2.13$ & 0.14 & $\mathrm{DyPO}_{4}$ \\
$\mathrm{Y}_{2} \mathrm{O}_{3}$ & 2.26 & $2.09-2.46$ & 0.13 & $\mathrm{YPO}_{4}$ \\
$\mathrm{Er}_{2} \mathrm{O}_{3}$ & 0.23 & $0.15-0.32$ & 0.07 & $\mathrm{ErPO}_{4}$ \\
$\mathrm{~Tb}_{2} \mathrm{O}_{3}$ & 0.44 & $0.35-0.51$ & 0.06 & $\mathrm{TbPO}_{4}$ \\
$\mathrm{Ho}_{2} \mathrm{O}_{3}$ & 0.12 & $0.00-0.20$ & 0.07 & $\mathrm{HoPO}_{4}$ \\
$\mathrm{UO}_{3}$ & 30.32 & $29.83-30.83$ & 0.31 & uranophane \\
$\mathrm{CO}_{2}$ (calc.) & $23.25 *$ & & & \\
$\mathrm{H}_{2} \mathrm{O}$ (calc.) & $20.06 *$ & & & \\
\hline total & 115.19 & & & \\
\hline
\end{tabular}

* calculated from the structure

$\mathrm{SD}$ - standard deviation 


\section{X-ray crystallography and structure determination}

A nearly triangular fragment of a tabular crystal of shabaite-( $\mathrm{Nd})$, with dimensions $0.12 \times 0.09 \times 0.02 \mathrm{~mm}$, was selected under the microscope and examined using an Rigaku/Oxford Diffraction Supernova single-crystal diffractometer with an Atlas S2 CCD detector using mirror-monochromatized $\mathrm{MoK}_{\alpha}$ radiation provided by the microfocus X-ray source. Shabaite-(Nd) is triclinic, space group $P-1$, with the unit-cell parameters $a=8.3835(5), b=9.2766(12)$, $c=31.7519(3) \AA, \alpha=90.058(3)^{\circ}, \beta=89.945$ $(4)^{\circ}, \gamma=90.331(4)^{\circ}$ and $V=2469.3(4) \AA^{3}$, $Z=4$. The diffraction pattern is strongly pseudomonoclinic, explaining the choice of $P 2, P m$ or $P 2 / m$ space groups by Deliens and Piret (1989). Data were finally integrated in the triclinic space group $P-1$; of the 26,331 collected reflections, 9676 were independent and 8441 were classified as observed based on the criterion $\left[I_{\mathrm{obs}}>\right.$ $3 \sigma(I)]$. Data were corrected for the Lorentz effect, polarization factor and background and for absorption by combining empirical scaling of the frames and Gaussian integration of the shape of the crystal in Jana2006 (Petříček et al. 2014). The $R_{\text {int }}$ of the dataset was 0.0405 . Other details of the data collection are given in Tab. 2.

The crystal structure of shabaite-(Nd) was solved by the charge-flipping algorithm of the Shelxt program (Sheldrick 2015) and subsequently refined using Jana2006 (Petř́čck et al. 2014) with the full-matrix least-squares refinement based on $F^{2}$. The first attempts to solve and refine the structure were done in monoclinic space groups; however, they did not lead to substantial success. Therefore, the triclinic centrosymmetric space group $P-1$ was chosen and twinning by reticular merohedry was introduced in to the refinement using the tool implemented in Jana2006 program (Petř́íček et al. 2016). The possibility of the acentric $P 1$ space group was also tested (including an inversion twin), however, it results in only slightly better final indices of agreement $(R \sim 5.8 \%)$, but having twice as many atoms in the unit-cell, many of them having nonpositively definite atomic displacement parameters, and large correlations of the fit overall. Finally, the solution and refinement in the centrosymmetric $P-1$ revealed the complete structure of shabaite-( $\mathrm{Nd})$, except the $\mathrm{H}$-atoms,
Tab. 2 Crystallographic data and refinement details for shabaite-(Nd)

\begin{tabular}{|c|c|}
\hline \multicolumn{2}{|l|}{ Crystal data } \\
\hline Formula & $\mathrm{Nd}_{2} \mathrm{Ca}\left[\left(\mathrm{UO}_{2}\right)\left(\mathrm{CO}_{3}\right)_{3}\right]\left(\mathrm{CO}_{3}\right)_{2}\left(\mathrm{H}_{2} \mathrm{O}\right)_{10.5}$ \\
\hline Crystal system & triclinic \\
\hline Space group & $P-1$ \\
\hline Unit-cell parameters: $a, b, c[\AA]$ & $8.3835(5), 9.2766(12), 31.7519(3)$ \\
\hline$\alpha, \beta, \gamma\left[^{\circ}\right]$ & $90.058(3), 89.945(4), 90.331(4)$ \\
\hline Unit-cell volume $\left[\AA^{3}\right]$ & $2469.3(4)$ \\
\hline $\mathrm{Z}$ & 4 \\
\hline Calculated density $\left[\mathrm{g} / \mathrm{cm}^{3}\right]$ & 2.926 \\
\hline Crystal size $[\mathrm{mm}]$ & $0.121 \times 0.091 \times 0.023$ \\
\hline \multicolumn{2}{|l|}{ Data collection } \\
\hline Diffractometer & Rigaku SuperNova with Atlas S2 CCD \\
\hline Temperature $[\mathrm{K}]$ & 297 \\
\hline Radiation, wavelength $[\AA]$ & $\mathrm{MoK}_{\alpha}, 0.71073(50 \mathrm{kV}, 30 \mathrm{~mA})$ \\
\hline$\theta$ range for data collection $\left[{ }^{\circ}\right]$ & $3.27-26.88$ \\
\hline Limiting Miller indices & $h=-10 \rightarrow 10, k=-11 \rightarrow 11, l=-38 \rightarrow 39$ \\
\hline Axis, frame width $\left({ }^{\circ}\right)$, time per frame $(\mathrm{s})$ & $\omega, 1.0,200$ \\
\hline Total reflections collected & 26331 \\
\hline Independent reflections & 9607 \\
\hline Independent observed reflections & $8435[I>3 \sigma(I)]$ \\
\hline Absorption coefficient $\left[\mathrm{mm}^{-1}\right]$, type & 11.01; Gaussian \\
\hline$T_{\min } / T_{\max }$ & $0.948 / 1.076$ \\
\hline$R_{\mathrm{int}}$ & 0.043 \\
\hline$F_{000}$ & 1928 \\
\hline Structure refinement by Jana2006 & Full-matrix least-squares on $F^{2}$ \\
\hline No. of ref. param., restraints, constraints & $339,0,0$ \\
\hline$R, w R$ (obs) & $0.0603,0.1238$ \\
\hline$R, w R$ (all) & $0.0695,0.1267$ \\
\hline GOF obs/all & $2.42,2.31$ \\
\hline Weighting scheme, weights & $\sigma, w=1 /\left(\sigma^{2}(I)+0.0004 I^{2}\right)$ \\
\hline Largest diff. peak and hole $\left(\mathrm{e}^{-} / \AA^{3}\right)$ & $-3.06,3.35$ \\
\hline Twin vol(1)/(2) & $0.8146(9), 0.1854(9)$ \\
\hline Twin matrix & $\left(\begin{array}{ccc}1 & 0 & 0 \\
0 & -1 & 0 \\
0 & 0 & -1\end{array}\right)$ \\
\hline
\end{tabular}

and converged to reasonable indices of agreement, $R=$ 0.0603 and $w R=0.1238$ for 8441 observed reflections with GOF $=2.42$ (Tab. 2). The atom coordinates and the displacement parameters are given in Tab. 3 and selected interatomic distances in Tab. 4. Bond-valence sums are shown in Tab. 5.

\subsection{Description of the crystal structure}

The structure of shabaite-(Nd) (Fig. 3) contains two $\mathrm{U}$, four $\mathrm{Nd}$, two $\mathrm{Ca}$, ten $\mathrm{C}$ and fifty-six $\mathrm{O}$ sites in the asymmetric unit. Each of two U sites, occupied by $\mathrm{U}^{6+}$, is coordinated by eight $\mathrm{O}$ atoms, two of which are strongly bonded by the triple $\mathrm{U}-\mathrm{O}$ bond $(\sim 1.79 \AA)$ forming the nearly linear uranyl ion, $\mathrm{UO}_{2}{ }^{2+}$, and six more are equatorially connected to form a hexagonal 
Tab. 3 Atom coordinates and displacement parameters for the crystal structure of shabaite-(Nd)

\begin{tabular}{|c|c|c|c|c|c|c|c|c|c|c|}
\hline Atom & $x / a$ & $y / b$ & $z / c$ & $U_{\text {eq }} / U_{\text {iso }}$ & $U^{11}$ & $U^{22}$ & $U^{33}$ & $U^{12}$ & $U^{13}$ & $U^{23}$ \\
\hline$\overline{\mathrm{U} 1}$ & $0.84141(8)$ & $0.38404(6)$ & $0.618431(19)$ & $0.01928(19)$ & $0.0235(4)$ & $0.0127(3)$ & $0.0216(3)$ & $-0.0017(3)$ & $-0.0002(3)$ & $0.0000(2)$ \\
\hline U2 & $0.34106(8)$ & $0.38452(6)$ & $0.881555(18)$ & $0.01949(19)$ & $0.0224(4)$ & $0.0157(3)$ & $0.0204(3)$ & $0.0004(3)$ & $0.0000(3)$ & $0.0018(2)$ \\
\hline Nd1 & $0.35528(10)$ & $0.39250(8)$ & $0.75179(3)$ & $0.0141(2)$ & $0.0080(4)$ & $0.0098(4)$ & $0.0243(5)$ & $-0.0034(3)$ & $-0.0005(4)$ & $0.0012(3)$ \\
\hline $\mathrm{Nd} 2$ & $-0.15000(10)$ & $0.89292(8)$ & $0.74560(3)$ & $0.0133(2)$ & $0.0081(4)$ & $0.0085(4)$ & $0.0234(5)$ & $-0.0027(4)$ & $0.0004(4)$ & $0.0000(3)$ \\
\hline $\mathrm{Nd} 3$ & $0.35006(10)$ & $0.89297(8)$ & $0.75444(3)$ & $0.0135(2)$ & $0.0086(4)$ & $0.0089(4)$ & $0.0229(5)$ & $-0.0030(4)$ & $-0.0008(4)$ & $0.0012(3)$ \\
\hline $\mathrm{Nd} 4$ & $-0.14489(10)$ & $0.39257(8)$ & $0.74826(3)$ & $0.0138(2)$ & $0.0071(4)$ & $0.0095(4)$ & $0.0246(5)$ & $-0.0028(3)$ & $0.0002(4)$ & $0.0001(3)$ \\
\hline Ca1 & $-0.2528(4)$ & $0.7503(4)$ & $0.55571(11)$ & $0.0257(11)$ & $0.030(2)$ & $0.0174(18)$ & $0.029(2)$ & $-0.0022(16)$ & $-0.0015(16)$ & $0.0000(14)$ \\
\hline $\mathrm{Ca} 2$ & $0.2470(4)$ & $0.7500(4)$ & $0.94430(11)$ & $0.0258(11)$ & $0.033(2)$ & $0.0177(18)$ & $0.027(2)$ & $-0.0016(16)$ & $0.0014(16)$ & $0.0000(14)$ \\
\hline $\mathrm{C} 1$ & $0.0394(17)$ & $0.1441(15)$ & $0.7722(4)$ & $0.009(3)^{*}$ & & & & & & \\
\hline $\mathrm{C} 2$ & $0.801(2)$ & $0.3831(18)$ & $0.5296(5)$ & $0.026(4) *$ & & & & & & \\
\hline C3 & $-0.3396(19)$ & $0.6441(15)$ & $0.7701(4)$ & $0.013(3)^{*}$ & & & & & & \\
\hline $\mathrm{C} 4$ & $-0.2369(17)$ & $0.6441(15)$ & $0.6644(5)$ & $0.013(3)^{*}$ & & & & & & \\
\hline $\mathrm{C} 5$ & $0.1600(18)$ & $0.6423(14)$ & $0.7302(4)$ & $0.009(3)^{*}$ & & & & & & \\
\hline C6 & $0.2660(18)$ & $0.6422(16)$ & $0.8355(5)$ & $0.015(3) *$ & & & & & & \\
\hline $\mathrm{C} 7$ & $0.4443(18)$ & $0.1408(17)$ & $0.8334(5)$ & $0.019(4) *$ & & & & & & \\
\hline $\mathrm{C} 8$ & $0.298(2)$ & $0.3831(17)$ & $0.9703(5)$ & $0.024(4) *$ & & & & & & \\
\hline C9 & $0.5396(17)$ & $0.1444(15)$ & $0.7282(4)$ & $0.009(3)^{*}$ & & & & & & \\
\hline $\mathrm{C} 10$ & $-0.0531(18)$ & $0.1379(16)$ & $0.6670(5)$ & $0.015(3) *$ & & & & & & \\
\hline O1 & $0.6420(14)$ & $0.3182(11)$ & $0.6246(3)$ & $0.027(3)^{*}$ & & & & & & \\
\hline $\mathrm{O} 2$ & $0.1104(12)$ & $0.2650(11)$ & $0.7697(3)$ & $0.020(2)^{*}$ & & & & & & \\
\hline $\mathrm{O} 3$ & $0.7788(13)$ & $0.4957(12)$ & $0.5540(4)$ & $0.029(3)^{*}$ & & & & & & \\
\hline $\mathrm{O} 4$ & $-0.2489(13)$ & $0.6320(11)$ & $0.6248(3)$ & $0.023(3) *$ & & & & & & \\
\hline $\mathrm{O} 5$ & $-0.4111(12)$ & $0.7642(11)$ & $0.7694(3)$ & $0.019(2)^{*}$ & & & & & & \\
\hline O6 & $0.0979(11)$ & $0.5176(10)$ & $0.7317(3)$ & $0.014(2)^{*}$ & & & & & & \\
\hline O7 & $0.6091(12)$ & $0.0214(10)$ & $0.7252(3)$ & $0.015(2) *$ & & & & & & \\
\hline O8 & $0.2532(12)$ & $0.7646(11)$ & $0.8170(3)$ & $0.020(2) *$ & & & & & & \\
\hline O9 & $0.4362(13)$ & $0.1416(12)$ & $0.8741(3)$ & $0.023(3)^{*}$ & & & & & & \\
\hline O10 & $-0.0286(12)$ & $0.0245(11)$ & $0.6867(3)$ & $0.020(2)^{*}$ & & & & & & \\
\hline O11 & $-0.2788(14)$ & $0.9289(12)$ & $0.6111(4)$ & $0.032(3) *$ & & & & & & \\
\hline O12 & $0.8473(15)$ & $0.2681(12)$ & $0.5504(4)$ & $0.035(3) *$ & & & & & & \\
\hline $\mathrm{O} 13$ & $-0.1115(12)$ & $0.1435(10)$ & $0.7707(3)$ & $0.015(2)^{*}$ & & & & & & \\
\hline $\mathrm{O} 14$ & $0.7887(15)$ & $0.6529(14)$ & $0.8707(4)$ & $0.041(3)^{*}$ & & & & & & \\
\hline O15 & $0.2206(14)$ & $0.9294(13)$ & $0.8885(4)$ & $0.033(3) *$ & & & & & & \\
\hline O16 & $-0.1234(15)$ & $0.4089(12)$ & $0.8242(4)$ & $0.037(3) *$ & & & & & & \\
\hline $\mathrm{O} 17$ & $0.6628(18)$ & $0.0459(14)$ & $0.9327(4)$ & $0.055(4) *$ & & & & & & \\
\hline O18 & $-0.2174(13)$ & $0.9143(12)$ & $0.8244(4)$ & $0.030(3)^{*}$ & & & & & & \\
\hline O19 & $0.3754(15)$ & $0.4068(13)$ & $0.6761(4)$ & $0.040(3)^{*}$ & & & & & & \\
\hline $\mathrm{O} 20$ & $0.7812(14)$ & $0.3867(12)$ & $0.4902(4)$ & $0.031(3)^{*}$ & & & & & & \\
\hline $\mathrm{O} 21$ & $0.2789(14)$ & $0.3868(12)$ & $0.0096(4)$ & $0.030(3)^{*}$ & & & & & & \\
\hline $\mathrm{O} 22$ & $0.2986(12)$ & $0.5232(11)$ & $0.8162(3)$ & $0.018(2)^{*}$ & & & & & & \\
\hline $\mathrm{O} 23$ & $-0.0658(17)$ & $1.0727(15)$ & $0.8835(4)$ & $0.053(4)^{*}$ & & & & & & \\
\hline $\mathrm{O} 24$ & $0.3169(12)$ & $0.6479(10)$ & $0.7297(3)$ & $0.014(2) *$ & & & & & & \\
\hline $\mathrm{O} 25$ & $0.5309(15)$ & $0.7634(14)$ & $0.9376(4)$ & $0.042(3)^{*}$ & & & & & & \\
\hline $\mathrm{O} 26$ & $0.2806(17)$ & $0.9848(15)$ & $0.9790(4)$ & $0.054(4)^{*}$ & & & & & & \\
\hline $\mathrm{O} 27$ & $0.0325(15)$ & $0.7614(14)$ & $0.5621(4)$ & $0.040(3)^{*}$ & & & & & & \\
\hline $\mathrm{O} 28$ & $0.2574(18)$ & $0.6844(17)$ & $1.0172(5)$ & $0.064(4)^{*}$ & & & & & & \\
\hline O29 & $0.1623(18)$ & $0.0468(14)$ & $0.5669(4)$ & $0.055(4)^{*}$ & & & & & & \\
\hline O30 & $-0.5296(17)$ & $0.7262(15)$ & $0.5583(4)$ & $0.055(4) *$ & & & & & & \\
\hline O31 & $0.4214(12)$ & $0.2619(11)$ & $0.8157(3)$ & $0.016(2)^{*}$ & & & & & & \\
\hline O32 & $1.0399(14)$ & $0.4465(13)$ & $0.6128(4)$ & $0.034(3)^{*}$ & & & & & & \\
\hline O33 & $0.2800(13)$ & $0.4952(12)$ & $0.9465(4)$ & $0.028(3)^{*}$ & & & & & & \\
\hline O34" & $0.924(3)$ & $-0.181(3)$ & $0.8819(8)$ & $0.042(7)^{*}$ & & & & & & \\
\hline O35 & $0.2838(13)$ & $0.9132(12)$ & $0.6759(3)$ & $0.027(3)^{*}$ & & & & & & \\
\hline O36 & $-0.4016(12)$ & $0.5180(10)$ & $0.7681(3)$ & $0.017(2)^{*}$ & & & & & & \\
\hline O37 & $0.1094(12)$ & $0.0201(10)$ & $0.7750(3)$ & $0.017(2)^{*}$ & & & & & & \\
\hline O38 & $0.3477(15)$ & $0.2695(12)$ & $0.9500(3)$ & $0.032(3) *$ & & & & & & \\
\hline O39 & $0.4351(17)$ & $0.0745(15)$ & $0.6168(4)$ & $0.053(4) *$ & & & & & & \\
\hline $\mathrm{O} 40$ & $0.1402(13)$ & $0.3184(11)$ & $0.8758(3)$ & $0.025(3)^{*}$ & & & & & & \\
\hline $\mathrm{O} 41$ & $-0.3897(12)$ & $0.2645(10)$ & $0.7304(3)$ & $0.017(2)^{*}$ & & & & & & \\
\hline $\mathrm{O} 42$ & $-0.2021(12)$ & $0.5230(11)$ & $0.6834(3)$ & $0.018(2) *$ & & & & & & \\
\hline $\mathrm{O} 43$ & $0.3889(12)$ & $0.1448(10)$ & $0.7294(3)$ & $0.015(2) *$ & & & & & & \\
\hline O44 & $-0.2458(12)$ & $0.7628(11)$ & $0.6833(3)$ & $0.019(2)^{*}$ & & & & & & \\
\hline $\mathrm{O} 45$ & $-0.1827(12)$ & $0.6477(10)$ & $0.7705(3)$ & $0.016(2) *$ & & & & & & \\
\hline O46 & $-0.0790(12)$ & $0.2616(11)$ & $0.6844(3)$ & $0.019(2)^{*}$ & & & & & & \\
\hline O47 & $0.5426(14)$ & $0.4461(13)$ & $0.8866(4)$ & $0.036(3) *$ & & & & & & \\
\hline O48 & $-0.2177(17)$ & $0.9826(15)$ & $0.5211(4)$ & $0.055(4) *$ & & & & & & \\
\hline O49 & $-0.0631(13)$ & $0.1394(12)$ & $0.6262(3)$ & $0.023(3)^{*}$ & & & & & & \\
\hline $\mathrm{O} 50$ & $0.0888(12)$ & $0.7620(11)$ & $0.7308(3)$ & $0.019(2)^{*}$ & & & & & & \\
\hline O51 & $0.2521(13)$ & $0.6302(12)$ & $0.8750(4)$ & $0.028(3) *$ & & & & & & \\
\hline O52 & $0.4732(12)$ & 0.0235(11) & $0.8132(3)$ & $0.020(2)^{*}$ & & & & & & \\
\hline O53 & $0.9711(17)$ & $-0.2716(15)$ & $0.9421(4)$ & $0.053(4)^{*}$ & & & & & & \\
\hline O54 & $-0.2465(18)$ & $0.6855(16)$ & $0.4832(5)$ & $0.063(4)^{*}$ & & & & & & \\
\hline O55 & $0.2902(15)$ & $0.6518(14)$ & $0.6294(4)$ & $0.042(3)^{*}$ & & & & & & \\
\hline O56 & $0.41(3)$ & $0.844(2)$ & $0.6190(7)$ & $0.028(6)^{*}$ & & & & & & \\
\hline
\end{tabular}

* - refined with isotropic atomic displacement parameters; \# - occupancy of 0.5 
Crystal structure of shabaite-(Nd)

bipyramid. Each of them are chelated by the three $\mathrm{CO}_{3}$ planar groups $(\mathrm{C} 2, \mathrm{C} 4, \mathrm{C} 10$ to $\mathrm{U} 1$ and $\mathrm{C} 6, \mathrm{C} 7, \mathrm{C} 8$ to U2) to form the well-known uranyl-tricarbonate complex (UTC), $\left[\left(\mathrm{UO}_{2}\right)\left(\mathrm{CO}_{3}\right)_{3}\right]^{4-}$. There are additional $\mathrm{CO}_{3}$ groups (C1, C3, C5 and C9) that are not linked to $\mathrm{U}$ sites, but are linked to the Nd-polyhedra. Each of the Nd sites is coordinated by nine ligands (Tab. 4) with an average distance of $\sim 2.47 \AA$, including $\mathrm{O}$ sites populated by $\mathrm{H}_{2} \mathrm{O}$ molecules. The $\mathrm{Ca}$ sites are regularly coordinated by seven ligands (Tab. 4); the Cal polyhedra are sharing a common edge with U1 hexagonal bipyramid and the remaining five ligands coordinated to the $\mathrm{Cal}$ site (defining regular pentagonal bipyramid) are $\mathrm{H}_{2} \mathrm{O}$ molecules, while the $\mathrm{Ca} 2$ site is linked in a similar way to the U2 hexagonal bipyramid.

With regard to the hierarchy of $\mathrm{U}^{6+}$ compounds developed by Burns and co-workers (Burns et al. 1996; Burns 2005; Lussier et al. 2016), the structure of shabaite-(Nd) is based upon finite clusters of polyhedra, the uranyltricarbonate clusters. Nevertheless, the structure of shabaite$(\mathrm{Nd})$ contains an interesting and dominant feature - infinite sheets of Nd-polyhedra interlinked by $\mathrm{CO}_{3}$ groups, stacked perpendicular to c (Fig. 3). These sheets have, overall, cationic character and consist of infinite chains of edge-sharing Nd-polyhedra that are running parallel to [100]. Adjacent chains are linked through $\mathrm{Nd}-\mathrm{O}$ and $\mathrm{C}-\mathrm{O}$ bonds, through the $\mathrm{CO}_{3}$ planar groups, which decorate the sheet (Fig. 4). Nearly perpendicularly to the plane of the sheet are staggered $\mathrm{CO}_{3}$ groups that are part of the UTC, and these also provide additional linkage with the sheet, through the apical O atoms of the Nd-polyhedra (Fig. 4). The remaining non-linked vertices of the Nd-polyhedra belong to the transformer $\mathrm{H}_{2} \mathrm{O}$ groups $(\mathrm{O} 16, \mathrm{O} 18, \mathrm{O} 19$, O35) (sensu Schindler and Hawthorne 2008), transforming and distributing bond-valence from the sheets
Tab. 4 Selected interatomic distances $(\AA)$ in the structure of shabaite-(Nd)

\begin{tabular}{|c|c|c|c|c|c|}
\hline $\mathrm{U} 1-\mathrm{O} 1$ & $1.787(11)$ & $\mathrm{U} 2-\mathrm{O} 40$ & $1.798(11)$ & Nd1-O2 & $2.431(10)$ \\
\hline U1-O32 & $1.768(12)$ & U2-O47 & $1.788(12)$ & Nd1-O6 & $2.539(10)$ \\
\hline U1-O3 & $2.356(11)$ & U2-O9 & $2.407(11)$ & Nd1-O19(Wa) & $2.412(12)$ \\
\hline $\mathrm{U} 1-\mathrm{O} 4{ }^{\mathrm{i}}$ & $2.434(11)$ & U2-O22 & $2.471(10)$ & $\mathrm{Nd} 1-\mathrm{O} 22$ & $2.424(10)$ \\
\hline U1-O12 & $2.413(11)$ & U2-O31 & $2.475(10)$ & $\mathrm{Nd} 1-\mathrm{O} 24$ & $2.495(9)$ \\
\hline $\mathrm{U} 1-\mathrm{O} 42^{\mathrm{i}}$ & $2.461(10)$ & U2-O33 & $2.360(11)$ & Nd1-O31 & $2.430(10)$ \\
\hline $\mathrm{U} 1-\mathrm{O} 46^{\mathrm{i}}$ & $2.477(10)$ & U2-O38 & $2.423(11)$ & Nd1-O36 ${ }^{\mathrm{i}}$ & 2.399(10) \\
\hline $\mathrm{U} 1-\mathrm{O} 49^{\mathrm{i}}$ & $2.423(11)$ & U2-O51 & $2.411(11)$ & $\mathrm{Nd} 1-\mathrm{O} 41^{\mathrm{i}}$ & $2.543(10)$ \\
\hline$<\mathrm{U} 1-\mathrm{O}_{U r}>$ & 1.778 & $<\mathrm{U} 2-\mathrm{O}_{U r}>$ & 1.793 & $\mathrm{Nd} 1-\mathrm{O} 43$ & $2.422(9)$ \\
\hline$<\mathrm{U} 1-\mathrm{O}_{\mathrm{eq}}>$ & 2.427 & $<\mathrm{U} 2-\mathrm{O}_{\mathrm{eq}}>$ & 2.425 & $<\mathrm{Nd} 1-\mathrm{O}>$ & 2.455 \\
\hline Nd2-O5 & $2.600(10)$ & $\mathrm{Nd} 3-\mathrm{O}^{\mathrm{i}}$ & $2.386(10)$ & $\mathrm{Nd} 4-\mathrm{O} 2$ & $2.545(10)$ \\
\hline $\mathrm{Nd} 2-\mathrm{O}^{\mathrm{ii}}$ & $2.440(10)$ & Nd3-O7 $7^{\text {iii }}$ & $2.640(10)$ & $\mathrm{Nd} 4-\mathrm{O} 6$ & $2.396(9)$ \\
\hline $\mathrm{Nd} 2-\mathrm{O} 10^{\mathrm{iii}}$ & $2.452(10)$ & Nd3-O8 & $2.453(10)$ & $\mathrm{Nd} 4-\mathrm{O} 13$ & $2.436(9)$ \\
\hline $\mathrm{Nd} 2-\mathrm{O} 13^{\mathrm{iii}}$ & $2.476(9)$ & $\mathrm{Nd} 3-\mathrm{O} 24$ & $2.419(9)$ & Nd4-O16(Wa) & $2.424(12)$ \\
\hline Nd2-O18(Wa) & $2.572(11)$ & Nd3-O35(Wa) & $2.561(11)$ & $\mathrm{Nd} 4-\mathrm{O} 36$ & $2.531(10)$ \\
\hline $\mathrm{Nd} 2-\mathrm{O} 37^{\mathrm{iii}}$ & $2.639(10)$ & $\mathrm{Nd} 3-\mathrm{O} 37^{\mathrm{iii}}$ & $2.432(10)$ & $\mathrm{Nd} 4-\mathrm{O} 41$ & $2.433(10)$ \\
\hline $\mathrm{Nd} 2-\mathrm{O} 44$ & $2.449(10)$ & $\mathrm{Nd} 3-\mathrm{O} 43^{\mathrm{iii}}$ & $2.488(9)$ & $\mathrm{Nd} 4-\mathrm{O} 42$ & $2.437(10)$ \\
\hline $\mathrm{Nd} 2-\mathrm{O} 45$ & $2.423(9)$ & Nd3-O50 & $2.610(10)$ & $\mathrm{Nd} 4-\mathrm{O} 45$ & $2.491(9)$ \\
\hline $\mathrm{Nd} 2-\mathrm{O} 50$ & $2.394(10)$ & $\mathrm{Nd} 3-\mathrm{O} 52^{\mathrm{iii}}$ & $2.449(10)$ & $\mathrm{Nd} 4-\mathrm{O} 46$ & $2.428(10)$ \\
\hline$<\mathrm{Nd} 2-\mathrm{O}>$ & 2.494 & $<\mathrm{Nd} 3-\mathrm{O}>$ & 2.493 & $<\mathrm{Nd} 4-\mathrm{O}>$ & 2.457 \\
\hline$\overline{\mathrm{Ca} 1-\mathrm{O} 3^{\mathrm{i}}}$ & $2.379(12)$ & $\mathrm{Ca} 2-\mathrm{O} 15(\mathrm{Wa})$ & $2.444(12)$ & $\mathrm{C} 1-\mathrm{O} 2$ & $1.269(17)$ \\
\hline $\mathrm{Ca} 1-\mathrm{O} 4$ & $2.454(11)$ & $\mathrm{Ca} 2-\mathrm{O} 25(\mathrm{Wa})$ & 2.392(13) & $\mathrm{C} 1-\mathrm{O} 13$ & $1.266(17)$ \\
\hline Ca1-O11(Wa) & $2.426(12)$ & $\mathrm{Ca} 2-\mathrm{O} 26(\mathrm{Wa})$ & $2.455(14)$ & $\mathrm{C} 1-\mathrm{O} 37$ & $1.297(17)$ \\
\hline $\mathrm{Ca} 1-\mathrm{O} 27(\mathrm{Wa})$ & $2.402(13)$ & $\mathrm{Ca} 2-\mathrm{O} 28(\mathrm{Wa})$ & $2.395(16)$ & $<\mathrm{C} 1-\mathrm{O}>$ & 1.277 \\
\hline $\mathrm{Ca} 1-\mathrm{O} 30(\mathrm{Wa})$ & 2.331(15) & $\mathrm{Ca} 2-\mathrm{O} 33$ & $2.383(12)$ & $\overline{\mathrm{C} 2-\mathrm{O} 3}$ & $1.31(2)$ \\
\hline Ca1-O48(Wa) & $2.437(15)$ & $\mathrm{Ca} 2-\mathrm{O} 51$ & $2.464(12)$ & $\mathrm{C} 2-\mathrm{O} 12$ & $1.32(2)$ \\
\hline $\mathrm{Ca} 1-\mathrm{O} 54(\mathrm{Wa})$ & $2.380(16)$ & $\mathrm{Ca} 2-\mathrm{O} 53^{\mathrm{ii}}(\mathrm{Wa})$ & $2.322(15)$ & $\mathrm{C} 2-\mathrm{O} 20$ & $1.26(2)$ \\
\hline$<\mathrm{Ca} 1-\mathrm{O}>$ & 2.401 & $<\mathrm{Ca} 2-\mathrm{O}>$ & 2.408 & $<\mathrm{C} 2-\mathrm{O}>$ & 1.297 \\
\hline C3-O5 & $1.268(17)$ & C4-O4 & $1.268(18)$ & $\mathrm{C} 5-\mathrm{O} 6$ & $1.266(16)$ \\
\hline $\mathrm{C} 3-\mathrm{O} 36$ & $1.279(17)$ & $\mathrm{C} 4-\mathrm{O} 42$ & $1.311(17)$ & $\mathrm{C} 5-\mathrm{O} 24$ & $1.316(18)$ \\
\hline $\mathrm{C} 3-\mathrm{O} 45$ & $1.315(19)$ & $\mathrm{C} 4-\mathrm{O} 44$ & $1.256(18)$ & $\mathrm{C} 5-\mathrm{O} 50$ & $1.264(17)$ \\
\hline$<\mathrm{C} 3-\mathrm{O}>$ & 1.29 & $<\mathrm{C} 4-\mathrm{O}>$ & 1.28 & $<\mathrm{C} 5-\mathrm{O}>$ & 1.28 \\
\hline C6-O8 & $1.284(18)$ & C7-O9 & $1.294(19)$ & $\mathrm{C} 8-\mathrm{O} 21^{\mathrm{x}}$ & $1.26(2)$ \\
\hline $\mathrm{C} 6-\mathrm{O} 22$ & $1.292(18)$ & $\mathrm{C} 7-\mathrm{O} 31$ & $1.273(18)$ & $\mathrm{C} 8-\mathrm{O} 33$ & $1.30(2)$ \\
\hline C6-O51 & $1.267(18)$ & $\mathrm{C} 7-\mathrm{O} 52$ & $1.286(18)$ & $\mathrm{C} 8-\mathrm{O} 38$ & $1.31(2)$ \\
\hline$<\mathrm{C} 6-\mathrm{O}>$ & 1.28 & $<\mathrm{C} 7-\mathrm{O}>$ & 1.28 & $<\mathrm{C} 8-\mathrm{O}>$ & 1.29 \\
\hline C9-O7 & $1.288(17)$ & $\mathrm{C} 10-\mathrm{O} 10$ & $1.242(18)$ & & \\
\hline C9-O41 $1^{\text {iv }}$ & $1.261(17)$ & $\mathrm{C} 10-\mathrm{O} 46$ & $1.292(18)$ & & \\
\hline C9-O38 & $1.31(2)$ & $\mathrm{C} 10-\mathrm{O} 49$ & $1.298(18)$ & & \\
\hline$<\mathrm{C} 9-\mathrm{O}>$ & 1.29 & $<\mathrm{C} 10-\mathrm{O}>$ & 1.28 & & \\
\hline
\end{tabular}

Symmetry codes: (i) $x+1, y, z$; (ii) $x-1, y+1, z$; (iii) $x, y+1, z$; (iv) $x-1, y, z$; (v) $x, y-1, z$; (vi) $x+1$, $y-1, z$; (vii) $-x+1,-y+1,-z+2$; (viii) $-x+1,-y+1,-z+1$; (ix) $-x,-y+1,-z+1$; (x) $x, y, z-1$; (xi) $-x+1$, $-y,-z+1$. Wa - molecular $\mathrm{H}_{2} \mathrm{O}$

to the UTC and Ca-polyhedra. The entire structure is therefore built upon electro-neutral sheets of composition $\left(\left\{\mathrm{Nd}^{3+}{ }_{2}\left(\mathrm{CO}_{3}\right)^{2-}{ }_{2}\left(\mathrm{H}_{2} \mathrm{O}\right)_{2}\right\}^{2+}\left\{\mathrm{Ca}\left(\mathrm{H}_{2} \mathrm{O}\right)_{5}\right\}^{2+}\left[\left(\mathrm{UO}_{2}\right)\right.\right.$ $\left.\left.\left(\mathrm{CO}_{3}\right)_{3}\right]^{4}\right)^{0}$, and between the adjacent sheets there are additional $\sim 3.5 \mathrm{H}_{2} \mathrm{O}$, which help to maintain the cohesion of structure though a network of H-bonds. The most prominent acceptors of the $\mathrm{H}$-bonds within the layers can be identified using the bond-valence analysis (Tab. 5). The formula obtained from the refinement is $\mathrm{Nd}_{2} \mathrm{Ca}\left[\left(\mathrm{UO}_{2}\right)\left(\mathrm{CO}_{3}\right)_{3}\right]\left(\mathrm{CO}_{3}\right)_{2}\left(\mathrm{H}_{2} \mathrm{O}\right)_{10.5}, \mathrm{Z}=4$. 


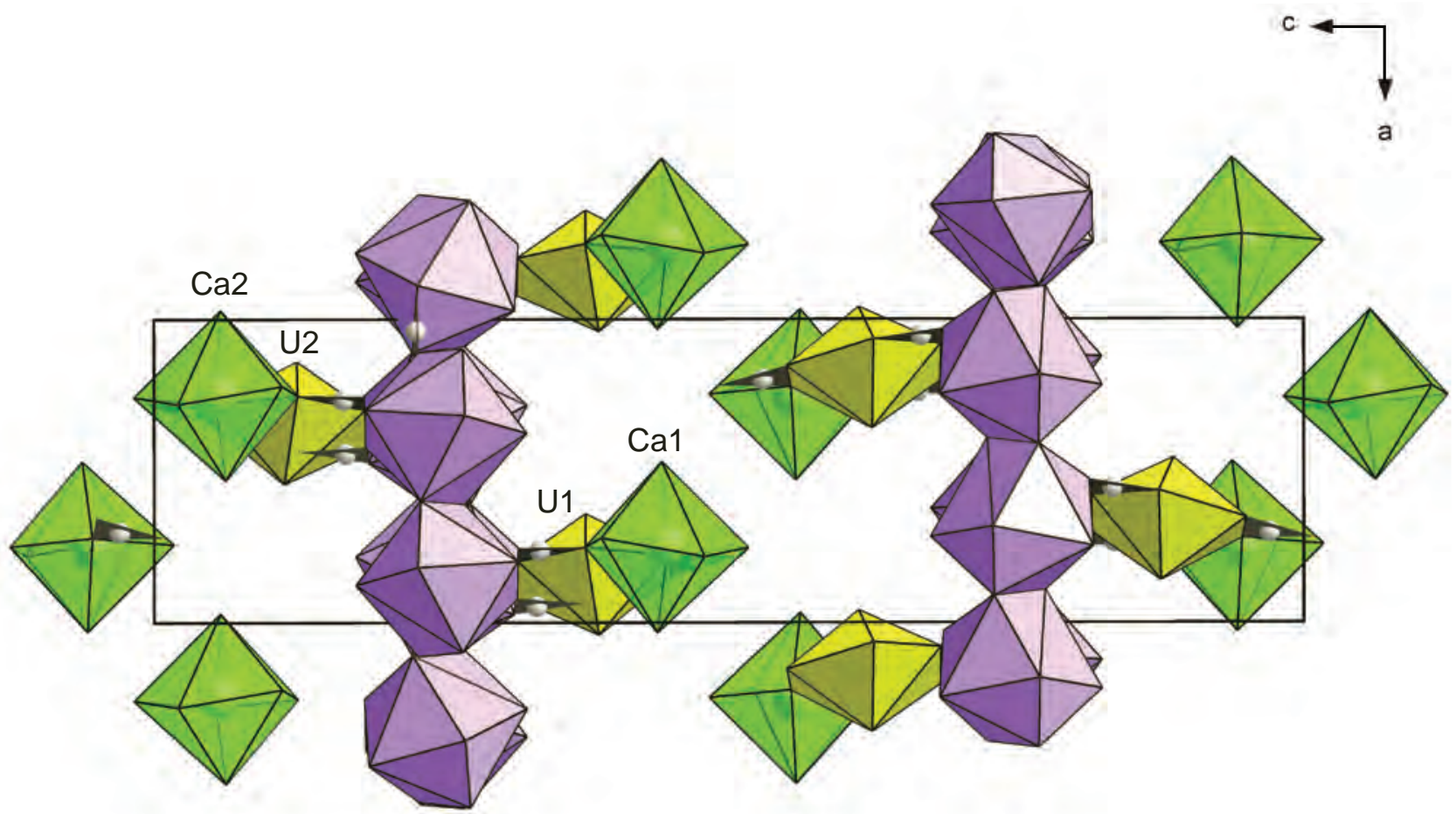

Fig. 3 The crystal structure of shabaite-(Nd) viewed down $\mathbf{b}$. Uranyl tricarbonate clusters (U-polyhedra in yellow and $\mathrm{CO}_{3}$ in black), are linked to infinite sheets of Nd-polyhedra (violet) stacked perpendicular to c. The Ca-polyhedra (green) are edge sharing with uranyl-tricarbonate complex (UTC). Molecules of $\mathrm{H}_{2} \mathrm{O}$ not bonded directly to any metal cation are omitted for clarity. Unit-cell edges are outlined by the solid black rectangle.

\section{Comments on original description}

Unit cell parameters $(a=9.208(5), b=32.09(3), c=$ $8.335(4) \AA$ and $\left.\beta=90.3(1)^{\circ}\right)$ and empirical formula of shabaite-(Nd) provided in the original description by

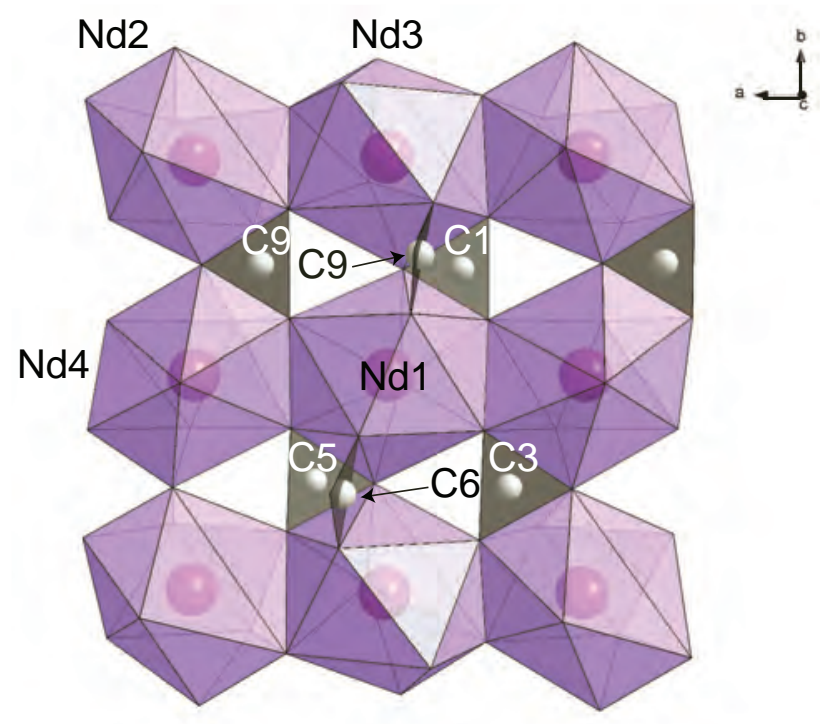

Fig. 4 Basic motif of the infinite Nd-sheet in the structure of shabaite$(\mathrm{Nd})$, composed of edge and corner sharing $\mathrm{Nd} \Phi_{9}$ polyhedra interlinked by $\mathrm{CO}_{3}$ groups $(\mathrm{C} 1, \mathrm{C} 3, \mathrm{C} 5$ and $\mathrm{C} 9)$; viewed down $\mathbf{c}$, slightly inclined for clarity. Through the $\mathrm{C} 6$ and $\mathrm{C} 9$ atoms the sheets are linked to the UTC clusters.
Deliens and Piret (1989) differ from those obtained by the current study. The unit cell parameters given in the original description refer to a monoclinic unit cell, which is a result of the twinning of the triclinic cell found in the current study. We also note that the physical properties (such as cleavage), optics, and descriptive morphology given in the original paper were based on the monoclinic unit cell settings, having the largest axis (in present study it is $\mathbf{c )}$ as a monoclinic $\mathbf{b}$.

Chemical composition inferred by Deliens and Piret (1989) was $\mathrm{Ca}(\mathrm{REE})_{2}\left(\mathrm{UO}_{2}\right)\left(\mathrm{CO}_{3}\right)_{4}(\mathrm{OH})_{2} \cdot 6 \mathrm{H}_{2} \mathrm{O}$ for $\mathrm{Z}=5$. The contents of $\mathrm{CO}_{2}$ and $\mathrm{H}_{2} \mathrm{O}$ ( $\mathrm{C}$ and $\mathrm{H}$, respectively) were obtained from the gas chromatography. The current crystal-structure study has revealed that there is an additional $\mathrm{CO}_{3}$ group linked to the Nd-polyhedra and there is no $\mathrm{OH}$ group within the structure. The formula introduced by the original description probably suffers from the low precision of the $\mathrm{C}$ and $\mathrm{H}$ determination, while using the low amounts of the analyzed sample.

\section{Relations to other uranyl carbonates}

Shabaite-(Nd) is one of the five known uranyl carbonates containing REE as an essential constituent. However, recent studies showed that bijvoetite-(Y) and kamotoite(Y) are likely the same species (Plášil and Petříček in print). Besides these two minerals, shabaite-(Nd) is the 
only (REE)-uranyl carbonate with a known structure. The structure of bijvoetite-(Y) (Li et al. 2000) and kamotoite-(Y) (Plášil and Petrríček 2016) is a sheet structure based upon the bijvoetite-(Y) uranyl-anion topology (Burns 2005; Lussier et al. 2016). In contrast, shabaite-( $\mathrm{Nd})$ contains finite clusters of polyhedra, the well-known UTC. There are probably substantial structural differences among the uranyl carbonates containing HREE and LREE or MREE. For example, kamotoite- $(\mathrm{Y}), \mathrm{Y}_{2}\left[\left(\mathrm{UO}_{2}\right)_{4} \mathrm{O}_{2}(\mathrm{OH})_{2}\left(\mathrm{CO}_{3}\right)_{4}\right]$ $\left(\mathrm{H}_{2} \mathrm{O}\right)_{13}$, does not contain any "lighter" metal cation as e.g. shabaite- $(\mathrm{Nd}), \mathrm{Nd}_{2} \mathrm{Ca}\left[\left(\mathrm{UO}_{2}\right)\left(\mathrm{CO}_{3}\right)_{3}\right]$ $\left(\mathrm{CO}_{3}\right)_{2}\left(\mathrm{H}_{2} \mathrm{O}\right)_{10.5}$. The structural unit of kamotoite-(Y) and bijvoetite-(Y) represents a higher degree of polymerization with $\mathrm{U}: \mathrm{C}$ ratio 1:1. We can only speculate about the unknown structure of e.g., astrocyanite- $(\mathrm{Ce})$, $\mathrm{Cu}_{2} \mathrm{Ce}_{2}\left(\mathrm{UO}_{2}\right)\left(\mathrm{CO}_{3}\right)_{5}(\mathrm{OH})_{2} \cdot 1.5 \mathrm{H}_{2} \mathrm{O}$ (Deliens and Piret 1990). However, based on similarities to shabaite$(\mathrm{Nd})$, and the presence of LREE, other metal cations and reported higher $\mathrm{U}: \mathrm{C}$ ratio, we can assume that it will also be a structure based upon finite clusters of polyhedra rather than with more polymerized structural units. Nevertheless, the structure of astrocyanite-(Ce) will probably be fairly unique owing to the importance of the Jahn-Teller effect on $\mathrm{Cu}^{2+}$ and the consequent specific impact on coordination environment around distorted $\mathrm{Cu}-\mathrm{O}$ polyhedra (Burns and Hawthorne 1995).

\section{Notice on origin of shabaite-( $\mathrm{Nd})$}

\subsection{Association of ( $\mathrm{Nd}$ )-rich minerals from Kamoto East}

Kamoto East open cut, Shaba, Congo, is a rich locality for several rare minerals that contain neodymium as an essential constituent. Besides here described shabaite-(Nd), it is francoisite- $(\mathrm{Nd}), \mathrm{Nd}\left[\left(\mathrm{UO}_{2}\right)_{3}\left(\mathrm{PO}_{4}\right)_{2} \mathrm{O}(\mathrm{OH})\right]\left(\mathrm{H}_{2} \mathrm{O}\right)_{6}$ (Piret et al. 1988), and schuilingite-(Nd), $\mathrm{PbCu}(\mathrm{Nd})$ $\left(\mathrm{CO}_{3}\right)_{3}(\mathrm{OH})\left(\mathrm{H}_{2} \mathrm{O}\right)$ (Vaes 1947; Schindler and Hawthorne 1999). Interestingly, the structure of schuilingite-(Nd) contains infinite chains of [9]-coordinated $\mathrm{Nd}^{3+}$-polyhedra, unlike francoisite-(Nd), where ${ }^{[9]} \mathrm{Nd}$ forms isolated polyhedra in interlayer of uranyl phosphate sheets. The frequency and relative abundance of $\mathrm{Nd}$ incorporated in minerals of supergene origin at Kamoto might suggest unusually high activity of $\mathrm{Nd}^{3+}$ in solutions from which this mineral formed. The fact that these phases are rare world-wide indicates that such a high $\mathrm{Nd}$ activity is otherwise uncommon at supergene conditions. Anyway this might be also due to the presence of specific source for $\mathrm{Nd}^{3+}$, i.e. it could have been be surrounding rocks rather than uraninite (see below).

\subsection{Rare earth elements in uranyl carbonate minerals}

Uranyl carbonates containing REE are rare alteration products of uraninite weathering in nature. Among them only kamotoite-(Y)/bijvoetite-(Y) has been reported from more than one locality. Astrocyanite-(Ce) and lepersonnite-(Gd) are known only from single localities: Kamoto East open cut (Deliens and Piret 1990) and Shinkolobwe mine (Deliens and Piret 1982), respectively. Lepersonnite-(Gd) at Shinkolobwe mine does not occur with any other (REE)-uranyl tricarbonate. It is usually associated with becquerelite, studtite and rarely with sharpite or urancalcarite-related minerals. Its chemical formula, $\mathrm{Ca}$ 


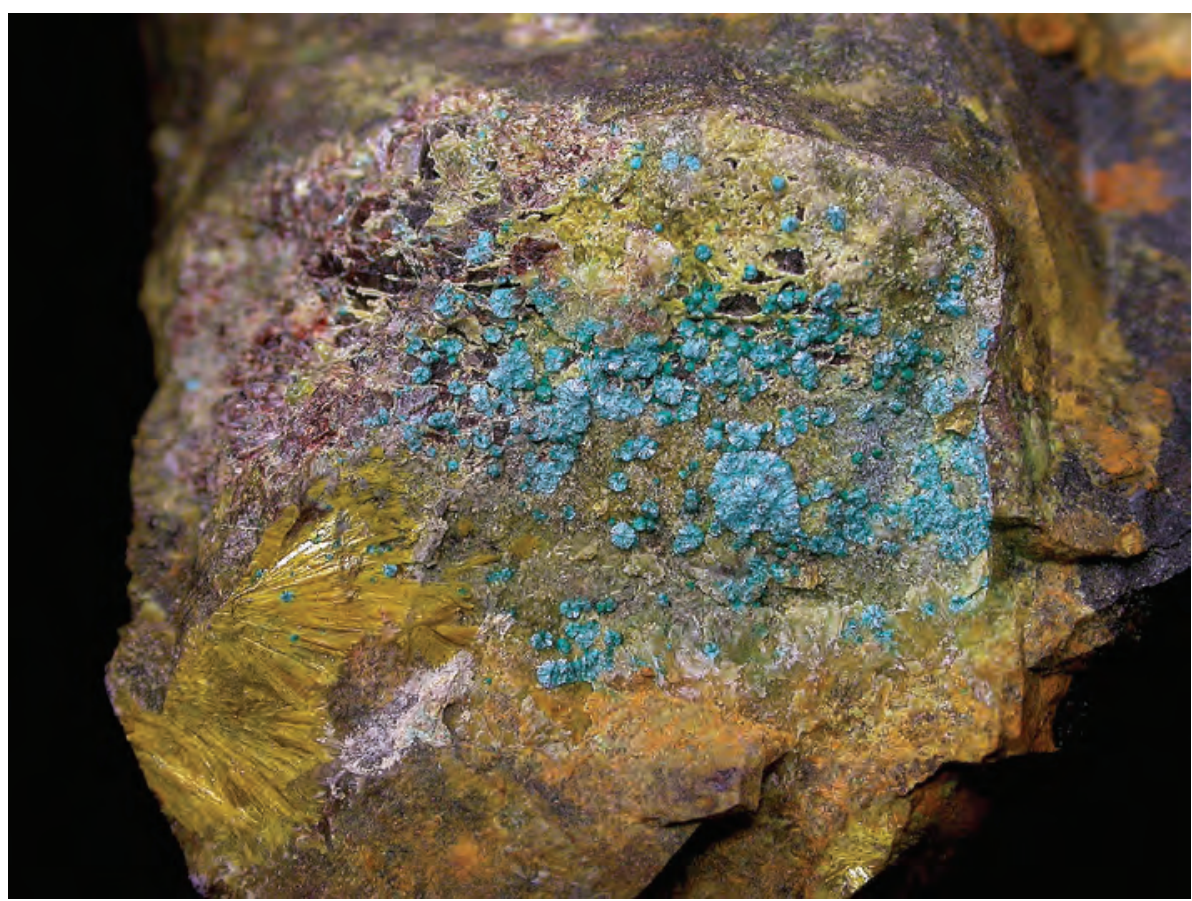

Fig. 5 Typical mineral association for Kamoto-East: bluish astrocyanite-(Ce) on-growing yellow kamotoite-( $\mathrm{Y})$ and also shabaite-(Nd) (beige to greenish white) on a fracture of strongly altered uraninite matrix with abundant vandendriesscheite (orange). Width of image is $4.2 \mathrm{~cm}$. Photo by Paul de Bondt.
$\mathrm{Gd}_{2}\left(\mathrm{UO}_{2}\right)_{24}\left(\mathrm{SiO}_{4}\right)_{4}\left(\mathrm{CO}_{3}\right)_{8}(\mathrm{OH})_{24} \cdot 48 \mathrm{H}_{2} \mathrm{O}$ (Deliens and Piret 1982), as well as uncommon mineral association, point towards its rarity and probably very special conditions that took place during the formation of this mineral.

Table 6 provides calculated chemical complexities of the known (REE)-uranyl carbonate minerals, showing that the exceptional chemical complexity (Siidra et al. 2014) of lepersonnite-(Gd) equals to to 508.88 bits/ fu. At Kamoto-East open cut, kamotoite-(Y), astrocyanite-(Ce) and shabaite-(Nd) occur. Shabaite-(Nd) is associated most frequently with astrocyanite-(Ce) and less also with kamotoite-( $\mathrm{Y})$; astrocyanite-(Ce) is usually clearly younger than kamotoite-(Y), on-growing its aggregates (Fig. 5). From Fig. 1 it is noticeable that shabaite-(Nd) overgrows older astrocyanite-(Ce). To the best of our knowledge, kamotoite-(Y) is nearly always overgrowing altered uraninite on samples from Kamoto. This is the case for shabaite-(Nd) as well, but its crystals do not necessarily sit directly on uraninite matrix.

We can only speculate about sources of the REE, without any additional detailed geochemical study, but in general, HREE (as Dy or Ho) and Y, usually concentrated

Tab. 6 Complexity measurements for the shabaite-(Nd) and related minerals

\begin{tabular}{lcc}
\hline Mineral & $I_{\mathrm{G}, \text { tot }}(\mathrm{bits} / \mathrm{cell})$ & chem $I$ (bits/fu) \\
\hline Shabaite-(Nd) & 918.99 & 99.22 \\
Astrocyanite-(Ce) & - & 52.13 \\
Lepersonnite-(Gd) & - & 508.88 \\
Kamotoite-(Y) & 1044.26 & 117.07 \\
\hline
\end{tabular}

calculations done after Krivovichev (2013) and Siidra et al. (2014) in primary uraninite (Janeczek and Ewing 1992), can be released during oxidation-hydration weathering (Finch and Ewing 1992; Göb et al. 2013; Plášil et al. 2014) and incorporated into the structure of kamotoite-(Y). The source of LREE may be in surrounding rocks and circulating fluids thus can play an important role during the formation of shabaite-( $\mathrm{Nd})$ and astrocyanite-(Ce).

Acknowledgements. The help of Jiří Čejka, who critically read the manuscript, is gratefully acknowledged. We thank Pavel Škácha and Paul de Bondt for microphotographs of shabaite-(Nd) and related phases. Jean-Claude Leydet is acknowledged for providing us material for study. The current text benefited from the constructive comments of two anonymous referees. František Laufek as well as editor-in-chief Vojtěch Janoušek are thanked for careful editorial handling of the manuscript. This research was supported by the project No. LO1603 under the Ministry of Education, Youth and Sports National sustainability program I of Czech Republic to JP.

Electronic supplementary material. Supplementary crystallographic data for this paper are available online at the Journal web site (http://dx.doi.org/10.3190/jgeosci.232)

\section{References}

Brown ID, Altermatt D (1985) Bond-valence parameters obtained from a systematic analysis of the inorganic crystal structure database. Acta Crystallogr B41: 244-248 
BURNs PC (2005) $\mathrm{U}^{6+}$ minerals and inorganic compounds: insights into an expanded structural hierarchy of crystal structures. Canad Mineral 43: 1839-1894

BuRns PC, HAWTHORNE FC (1995) Coordination-geometry structural pathways in $\mathrm{Cu}^{2+}$ oxysalt minerals. Canad Mineral 33: 8890-905

Burns PC, Miller ML, Ewing RC (1996) $\mathrm{U}^{6+}$ minerals and inorganic phases: a comparison and hierarchy of crystal structures. Canad Mineral 34: 845-880

Burns PC, Ewing RC, Hawthorne FC (1997) The crystal chemistry of hexavalent uranium: polyhedron geometries, bond-valence parameters, and polymerization of polyhedra. Canad Mineral 35: 1551-1570

Deliens M, PiRet P (1982) Bijvoetite et lepersonnite, carbonates hydrates d'uranyle et de terres rares de Shinkolobwe, Zaïre. Canad Mineral 20: 231-238

Deliens M, Piret P (1986) La kamotoïte-(Y), un nouveau carbonate d'uranyle et de terres rares de Kamoto, Shaba, Zaïre. Bull Soc franc Minéral Cristallogr 109: 643-647

Deliens M, Piret P (1989) La shabaite-(Nd), $\mathrm{Ca}(T R)_{2}\left(\mathrm{UO}_{2}\right)$ $\left(\mathrm{CO}_{3}\right)_{4}(\mathrm{OH})_{2} \cdot 6 \mathrm{H}_{2} \mathrm{O}$, nouvelle espèce minérale de Kamoto, Shaba, Zaire. Eur J Mineral 1: 85-88

Deliens M, Piret P (1990) L'astrocyanite-(Ce), $\mathrm{Cu}_{2}(\mathrm{TR})_{2}\left(\mathrm{UO}_{2}\right)\left(\mathrm{CO}_{3}\right)_{5}(\mathrm{OH})_{2} \cdot 1.5 \mathrm{H}_{2} \mathrm{O}$, nouvelle espèce minérale de Kamoto, Shaba, Zaïre. Eur J Mineral 2: 407-411

Finch RJ, EwING RC (1992) The corrosion of uraninite under oxidizing conditions. J Nucl Mater 190: 133-156

FinCH RJ, MuRAKAMI T (1999) Systematics and paragenesis of uranium minerals. In: BuRNs PC, FINCH RJ (eds) Uranium: Mineralogy, Geochemistry and the Environment. Mineralogical Society of America and Geochemical Society Reviews in Mineralogy and Geochemistry 38: pp 91-179

GAGNÉ OC, HaWtHORNe FC (2015) Comprehensive derivation of bond-valence parameters for ion pairs involving oxygen. Acta Crystallogr B71: 562-578

Göв S, Guhring JE, Bau M, MarkL G (2013) Remobilization of $U$ and REE and the formation of secondary minerals in oxidized U deposits. Amer Miner 98: 530-548

JANECZEK J, EwING RC (1992) Structural formula of uraninite. J Nucl Mater 190: 128-132

KRIVOVICHEV SV (2013) Structural complexity of minerals: information storage and processing in the mineral world. Mineral Mag 77: 275-326

KrIVovichev SV, PLÁŠIL J (2013) Mineralogy and crystallography of uranium. In: BuRns PC, SigmON GE (eds) Uranium: From Cradle to Grave. Mineralogical Association of Canada Short Courses 43: pp 15-119
LANGMUIR D (1978) Uranium solution-mineral equilibria at low temperatures with applications to sedimentary ore. Geochim Cosmochim Acta 42: 547-569

Li Y, BuRns PC, GAULT RA (2000) A new rare-earth-element uranyl carbonate sheet in the structure of bijvoetite. Canad Mineral 38: 153-162

LusSiER AJ, LOPEZ RAK, Burns PC (2016) A revised and expanded structure hierarchy of natural and synthetic hexavalent uranium compounds. Canad Mineral 54: 177-283

Petř́iček V, Dušek M, Palatinus L (2014) Crystallographic computing system JANA2006: general features. Z Kristallogr 229: 345-352

Petř́ ÍčeK V, DušeK M, PlášIl J (2016) Crystallographic computing system Jana2006: solution and refinement of twinned structures. Z Kristallogr 231: 583-599

Piret P, Deliens M, Piret-Meunier J (1988) La françoisite(Nd), nouveau phosphate d'uranyle et de terres rares; propriétés et structure cristalline. Bull Minéral 111: 443-449

PLÁŠIL J (2014) Oxidation-hydration weathering of uraninite: the current state-of-knowledge. J Geosci 59: 99-114

Plášsl J, Petréičé V (in print) Crystal structure of the (REE)-uranyl carbonate mineral kamotoite-(Y). Mineral Mag, doi: 10.1180/minmag.2016.080.123

Plášil J, SeJKora J, ŠKoda R, ŠKácha P (2014) The recent weathering of uraninite from the Červená vein, Jáchymov (Czech Republic): a fingerprint of the primary minerals geochemistry in the alteration association. J Geosci 59: 223-253

Pouchou JL, Pichoir F (1985) “PAP” ( $\varphi \rho Z)$ procedure for improved quantitative microanalysis. In: ARMSTRONG JT (ed) Microbeam Analysis. San Francisco Press, San Francisco, pp 104-106

SCHINDLER M, HaWthorNe FC (1999) The crystal structure of schuilingite-(Nd). Canad Mineral 37: 1463-1470

SCHINDLER M, Hawthorne FC (2008) The stereochemistry and chemical composition of interstitial complexes in uranyl-oxysalt minerals. Canad Mineral 46: 467-501

SHELDRICK GM (2015) SHELXT - integrated space-group and crystal-structure determination. Acta Crystallogr A71: 3-8

Siidra O, Zenko DS, Krivovichev SV (2014) Structural complexity of lead silicates: crystal structure of $\mathrm{Pb}_{21}\left[\mathrm{Si}_{7} \mathrm{O}_{22}\right]_{2}\left[\mathrm{Si}_{4} \mathrm{O}_{13}\right]$ and its comparison to hyttsjöite. Amer Miner 99: 817-823

VAES JF (1947) Six nouveaux minéraux d'urane provenant de Shinkolobwe (Katanga). Annal Soc Géol Belg 70: 212-225 
